Publisher: Research Council of Science and Technology, Biratnagar, Nepal:

p. 80

\title{
BIBECHANA
}

A Multidisciplinary Journal of Science, Technology and Mathematics

ISSN 2091-0762 (print), 2382-5340 (online)

Journal homepage: http://nepjol.info/index.php/BIBECHANA

\section{On contact conformal curvature tensor in trans-Sasakian manifolds}

\author{
Riddhi Jung Shah \\ Department of Mathematics, Janata Campus \\ Nepal Sanskrit University, Dang \\ E-mail: shahrigeo@gmail.com \\ Accepted for publication: December 18, 2014
}

\begin{abstract}
The purpose of this paper is to study some results on contact conformal curvature tensor in trans-Sasakian manifolds. Contact conformally flat trans-Sasakian manifold, $\xi$-contact conformally flat trans-Sasakian manifold and curvature conditions $C_{0}(\xi, X) \cdot S=0$ and $C_{0}(\xi, X) \cdot C_{0}=0$ are studied with some interesting results. Finally, we study an example of 3dimensional trans-Sasakian manifold.

DOI: http://dx.doi.org/10.3126/bibechana.v12i0.11783

(C) 2014 RCOST: All rights reserved.
\end{abstract}

Keywords: Contact conformal curvature tensor; Trans-Sasakian manifold; Hermitian manifolds.

\section{Introduction}

In 1978, Gray and Hervella [1] studied on the sixteen classes of almost Hermitian manifolds and their linear invariants. They considered unitary group $U(n)$ on a certain space $W$ and studied that the representation of $U(n)$ on $W$ has four irreducible components, $W=W_{1} \oplus W_{2} \oplus W_{3} \oplus W_{4}$. From these four components sixteen different invariants subspaces were obtained. Among four components $W_{3} \oplus W_{4}$ corresponds to the class of Hermitian manifolds. Oubina [2] studied a new class of almost contact metric structure, called trans-Sasakian which is an analogue of a locally conformal Kaehler structure on an almost Hermitian manifold. An almost contact metric structure $(\varphi, \xi, \eta, g)$ (where $\varphi$ is a $(1,1)$ tensor field, $\xi$ is a vector field, $\eta$ is a 1 -form and $g$ is a compatible Rimannian metric) on $M$ is trans-Sasakian [2] if $(M \times \mathbb{R}, J, G)$ belongs to the class $W_{4}$, where $J$ is the almost complex structure on $M \times \mathbb{R}$ defined by

$$
J\left(X, f \frac{d}{d t}\right)=\left(\varphi X-f \xi, \eta(X) \frac{d}{d t}\right),
$$


Shah/BIBECHANA 12 (2015) 80-88: p. 81

for any vector field $X$ on $M$, where $G$ is the product metric on $M \times \mathbb{R}$. Trans-Sasakian manifold is the trans-Sasakian structure of type $(\alpha, \beta)$, where $\alpha$ and $\beta$ are smooth functions on $M$. TransSasakian manifolds of type $(0,0),(\alpha, 0)$ and $(0, \beta)$ are cosympletic [3], $\alpha$-Sasakian [4] and $\beta$ Kenmotsu manifold $[4,5]$ respectively. Trans-Sasakian manifolds have been studied in $[6,7]$ and by many others.

On the other hand, contact conformal curvature tensor field was introduced and defined by Jeong et al. [8] in a $(2 n+1)$-dimensional Sasakian manifold which was constructed from the conformal curvature tensor field defined by Kitahara et al. [9] in a Kaehler manifold by using the BoothbyWang's fibration. Contact conformal curvature tensor has also been studied in [10] and [11].

\section{Preliminaries}

Let $M$ be a $(2 n+1)$-dimensional almost contact metric manifold equipped with an almost contact metric structure $(\varphi, \xi, \eta, g)$, where $\varphi$ is a $(1,1)$ tensor field, $\xi$ is a vector field, $\eta$-is a 1 -form and $g$ is a compatible Riemannian metric such that [3]

$$
\begin{gathered}
\varphi^{2}(X)=-X+\eta(X) \xi, \eta(\xi)=1, \varphi \xi=0, \eta(\varphi X)=0, \\
g(\varphi X, \varphi Y)=g(X, Y)-\eta(X) \eta(Y), \\
g(\varphi X, Y)=-g(X, \varphi Y), g(X, \xi)=\eta(X),
\end{gathered}
$$

for all $X, Y \in T M$. The fundamental 2-form $\Phi$ of the almost contact metric structure $(\varphi, \xi, \eta, g)$ is defined as

$$
\Phi(X, Y)=g(X, \varphi Y)=-g(\varphi X, Y),
$$

since $\varphi$ is a skew-symmetric with respect to $g$.

An almost contact metric manifold $M$ is called trans-Sasakian manifold if [2]

(2.5) $\left(\nabla_{X} \varphi\right) Y=\alpha\{g(X, Y) \xi-\eta(Y) X\}+\beta\{g(\varphi X, Y) \xi-\eta(Y) \varphi X\}$,

where $\nabla$ is Levi-Civita connection of Riemannian metric $g$ and $\alpha, \beta$ are smooth functions on $M$.

From (2.5) it follows that

$$
\begin{gathered}
\nabla_{X} \xi=-\alpha \varphi X+\beta\{X-\eta(X) \xi\}, \\
\left(\nabla_{X} \eta\right) Y=-\alpha g(\varphi X, Y)+\beta g(\varphi X, \varphi Y) .
\end{gathered}
$$

In a $(2 n+1)$-dimensional trans-Sasakian manifold $M$, the following relations hold [6]

$$
\begin{aligned}
R(X, Y) \xi= & \left(\alpha^{2}-\beta^{2}\right)[\eta(Y) X-\eta(X) Y]-(X \alpha) \varphi Y-(X \beta) \varphi^{2}(Y) \\
& +2 \alpha \beta[\eta(Y) \varphi X-\eta(X) \varphi Y]+(Y \alpha) \varphi X+(Y \beta) \varphi^{2}(X), \\
R(\xi, X) Y= & \left(\alpha^{2}-\beta^{2}\right)[g(X, Y) \xi-\eta(Y) X]+2 \alpha \beta[g(\varphi Y, X) \xi \\
& -\eta(Y) \varphi X]+(Y \alpha) \varphi X+g(\varphi Y, X)(\operatorname{grad} \alpha) \\
& +(Y \beta)[X-\eta(X) \xi]-g(\varphi X, \varphi Y)(\operatorname{grad} \beta),
\end{aligned}
$$




$$
\begin{gathered}
2 \alpha \beta+(\xi \alpha)=0, \\
S(X, \xi)=\left[2 n\left(\alpha^{2}-\beta^{2}\right)-(\xi \beta)\right] \eta(X)-((\varphi X) \alpha)-(2 n-1)(X \beta), \\
\eta(R(X, Y) Z)=-g(R(X, Y) \xi, Z), \\
\eta(R(X, Y) \xi)=\eta(R(\xi, X) \xi)=\eta(R(X, \xi) \xi)=0, \\
\eta(R(\xi, X) Y)=\left(\alpha^{2}-\beta^{2}-(\xi \beta)\right) g(\varphi X, \varphi Y) .
\end{gathered}
$$

In a $(2 n+1)$-dimensional trans-Sasakian manifold if we put $\varphi(\operatorname{grad} \alpha)=(2 n-1) \operatorname{grad} \beta$, then we have

$$
\begin{gathered}
(\xi \beta)=0, \\
S(X, \xi)=2 n\left(\alpha^{2}-\beta^{2}\right) \eta(X), \\
\eta(R(\xi, X) Y)=\left(\alpha^{2}-\beta^{2}\right) g(\varphi X, \varphi Y), \\
R(\xi, X) \xi=\left(\alpha^{2}-\beta^{2}\right)\{\eta(X) \xi-X\}, \\
R(X, \xi) \xi=-R(\xi, X) \xi .
\end{gathered}
$$

Throughout the paper we consider the trans-Sasakian manifold under the condition $\varphi(\operatorname{grad} \alpha)=(2 n-1) \operatorname{grad} \beta$.

In a $(2 n+1)$-dimensional trans-Sasakian manifold the contact conformal curvature tensor field $C_{0}$ of type $(1,3)$ which is defined by [8] can be written as

$$
\begin{aligned}
C_{0}(X, Y) Z= & R(X, Y) Z+\frac{1}{2 n}\{S(Y, Z) X-S(X, Z) Y+g(Y, Z) Q X \\
& -g(X, Z) Q Y+S(X, Z) \eta(Y) \xi-S(Y, Z) \eta(X) \xi \\
& +\eta(X) \eta(Z) Q Y-\eta(Y) \eta(Z) Q X+S(\varphi X, Z) \varphi Y \\
& -S(\varphi Y, Z) \varphi X+g(X, \varphi Z) Q(\varphi Y)-g(Y, \varphi Z) Q(\varphi X) \\
& +2 g(X, \varphi Y) Q(\varphi Z)+2 S(\varphi X, Y) \varphi Z\} \\
& +\frac{1}{2 n(n+1)}\left\{2 n^{2}-n-2+\frac{(n+2) r}{2 n}\right\}\{g(Y, \varphi Z) \varphi X \\
& -g(X, \varphi Z) \varphi Y-2 g(X, \varphi Y) \varphi Z\} \\
& +\frac{1}{2 n(n+1)}\left\{n+2-\frac{(3 n+2) r}{2 n}\right\}\{g(Y, Z) X-g(X, Z) Y\} \\
- & \frac{1}{2 n(n+1)}\left\{4 n^{2}+5 n+2-\frac{(3 n+2) r}{2 n}\right\}\{\eta(Y) \eta(Z) X \\
- & \eta(X) \eta(Z) Y+g(Y, Z) \eta(X) \xi-g(X, Z) \eta(Y) \xi\},
\end{aligned}
$$

where $R, S, Q$ and $r$ denote the curvature tensor, the Ricci tensor, the Ricci operator and the scalar curvature respectively.

From (2.20), we also have 


$$
\begin{gathered}
C_{0}(X, Y) \xi=R(X, Y) \xi+\left(\alpha^{2}-\beta^{2}-2\right)\{\eta(Y) X-\eta(X) Y\}, \\
\begin{aligned}
& C_{0}(\xi, X) Y=R(\xi, X) Y+\left(\alpha^{2}-\beta^{2}-2\right)\{g(X, Y) \xi-\eta(Y) X\}, \\
& \eta\left(C_{0}(X, Y) Z\right)= \eta(R(X, Y) Z) \\
&+\left(\alpha^{2}-\beta^{2}-2\right)\{g(Y, Z) \eta(X)-g(X, Z) \eta(Y)\}, \\
& \eta\left(C_{0}(X, Y) \xi\right)=0,
\end{aligned} \\
\eta\left(C_{0}(\xi, X) Y\right)=2\left(\alpha^{2}-\beta^{2}-1\right)\{g(X, Y)-\eta(X) \eta(Y)\} .
\end{gathered}
$$

Definition. A $(2 n+1)$-dimensional trans-Sasakian manifold $M$ is said to be an $\eta$-Einstein manifold if its Ricci tensor $S$ of type $(0,2)$ is of the form

$$
S(X, Y)=a g(X, Y)+b \eta(X) \eta(Y),
$$

where $a, b$ are smooth functions on $M$. If $b=0$, then the manifold $M$ becomes an Einstein manifold.

\section{Contact Conformally Flat Trans-Sasakian Manifold}

Definition. A $(2 n+1)$-dimensional trans-Sasakian manifold is said to be contact conformally flat if it satisfies the condition

$$
C_{0}(X, Y) Z=0
$$

Now, we prove the following result:

Theorem 3.1. If a $(2 n+1)$-dimensional trans-Sasakian manifold $M$ is contact conformally flat, then $\alpha^{2}=\beta^{2}+1$.

Proof. Let $M$ be a $(2 n+1)$-dimensional trans-Sasakian manifold. Suppose $M$ is contact conformally flat then the condition $C_{0}(X, Y) Z=0$ holds. Now, using (3.1) in (2.20) and taking inner product on both sides by $\xi$, we get

$$
\begin{aligned}
\eta(R(X, Y) Z)= & \frac{1}{2 n}[g(X, Z) S(Y, \xi)-g(Y, Z) S(X, \xi) \\
& -\eta(X) \eta(Z) S(Y, \xi)+\eta(Y) \eta(Z) S(X, \xi)] \\
& +2[g(Y, Z) \eta(X)-g(X, Z) \eta(Y)] .
\end{aligned}
$$

In view of (2.12), (2.16) and (3.2), we get

$$
\begin{aligned}
0 & =2\left(\alpha^{2}-\beta^{2}-1\right)[g(Y, Z) \eta(X)-g(X, Z) \eta(Y)]+2 \alpha \beta[g(\varphi Y, Z) \eta(X) \\
& -g(\varphi X, Z) \eta(Y)]+(X \alpha) g(\varphi Y, Z)-(Y \alpha) g(\varphi X, Z)-(X \beta) g(\varphi Y, \varphi Z) .
\end{aligned}
$$

Putting $X=\xi$ in (3.3) and using (2.1), (2.10) and (2.15), we obtain

$$
\left(\alpha^{2}-\beta^{2}-1\right)[g(Y, Z)-\eta(Y) \eta(Z)]=0 .
$$

Since $g(Y, Z)-\eta(Y) \eta(Z) \neq 0$, we have $\left(\alpha^{2}-\beta^{2}-1\right)=0$. This implies that 


$$
\alpha^{2}=\beta^{2}+1
$$

This completes the proof of the theorem.

\section{4. $\xi$-Contact Conformally Flat Trans-Sasakian Manifold}

Definition. A trans-Sasakian manifold of dimension $(2 n+1)$ is said to be $\xi$-contact conformally flat if the condition

$$
C_{0}(X, Y) \xi=0
$$

holds.

Theorem 4.1. Let $M$ be a $(2 n+1)$-dimensional trans-Sasakian manifold satisfying the condition $C_{0}(X, Y) \xi=0$, then $\alpha^{2}=\beta^{2}+1$.

Proof. Let us consider a $(2 n+1)$-dimensional trans-Sasakian manifold $M$ which satisfies the condition $C_{0}(X, Y) \xi=0$. Then by virtue of (2.1), (2.3), (2.8), (2.16) and (4.1) in (2.20), we get

$$
\begin{aligned}
0 & =2\left(\alpha^{2}-\beta^{2}-1\right)\{\eta(Y) X-\eta(X) Y\}-(X \alpha) \varphi Y+(X \beta) Y \\
& -(X \beta) \eta(Y) \xi+2 \alpha \beta[\eta(Y) \varphi X-\eta(X) \varphi Y]+(Y \alpha) \varphi X \\
& -(Y \beta) X+(Y \beta) \eta(X) \xi .
\end{aligned}
$$

Putting $X=\xi$ in (4.2) and using (2.1), (2.10) and (2.15), we obtain

$$
\left(\alpha^{2}-\beta^{2}-1\right)\{\eta(Y) \xi-Y\}=0 \text {. }
$$

Since $\eta(Y) \xi-Y=\varphi^{2}(Y) \neq 0$, we have $\left(\alpha^{2}-\beta^{2}-1\right)=0$. This yields

$$
\alpha^{2}=\beta^{2}+1 \text {. }
$$

Thus the theorem is proved.

From theorem 3.1 and theorem 4.1, we can state the following result:

Theorem 4.2. Trans-Sasakian manifolds of dimension $(2 n+1)$ which satisfy the conditions $C_{0}(X, Y) Z=0$ and $C_{0}(X, Y) \xi=0$ are equivalent.

\section{Trans-Sasakian Manifold Satisfying $\mathrm{C}_{0}(\xi, X) . S=0$}

Consider a trans-Sasakian manifold $M$ of dimension $(2 n+1)$. Let $S$ be the Ricci tensor of type ( 0 , 2). We prove the following result:

Theorem 5.1. Let $M$ be a $(2 n+1)$-dimensional trans-Sasakian manifold. If $M$ satisfies the condition $C_{0}(\xi, X) . S=0$, then it is an Einstein manifold with scalar curvature $r=2 n(2 n+1)\left(\alpha^{2}-\beta^{2}\right)$.

Proof. Let $M$ be a $(2 n+1)$-dimensional trans-Sasakian manifold which satisfies the condition 


$$
C_{0}(\xi, X) \cdot S(U, V)=0
$$

This condition implies that

$$
S\left(C_{0}(\xi, X) U, V\right)+S\left(U, C_{0}(\xi, X) V\right)=0 .
$$

Putting $V=\xi$ in (5.2) and using (2.16) and (2.22), we obtain

$$
S(X, U)=2 n\left(\alpha^{2}-\beta^{2}\right) g(X, U) .
$$

Taking an orthonormal frame field at any point of the manifold and contracting over $X$ and $U$ in (5.3), we get

$$
r=2 n(2 n+1)\left(\alpha^{2}-\beta^{2}\right) .
$$

From (5.3) and (5.4) it follows that the manifold $M$ is an Einstein manifold with scalar curvature $r=2 n(2 n+1)\left(\alpha^{2}-\beta^{2}\right)$. This completes the proof of the result.

\section{Trans-Sasakian Manifold Satisfying $C_{0}(\xi, X) \cdot C_{0}=0$}

Let $M$ be a $(2 n+1)$-dimensional trans-Sasakian manifold. Suppose the condition $\left(C_{0}(\xi, X) \cdot C_{0}\right)(U, V) Z=0$ holds in $M$. Then we have

Theorem 6.1. A $(2 n+1)$-dimensional trans-Sasakian manifold satisfying the condition $C_{0}(\xi, X) \cdot C_{0}=0$ is contact conformally semi-symmetric if

$$
\begin{aligned}
0= & \left(\alpha^{2}-\beta^{2}\right)\{2 g(V, Z) X-g(X, V) Z-g(X, Z) V\} \\
& -g(R(\xi, V) Z, X) \xi-R(X, V) Z .
\end{aligned}
$$

Proof. Let us consider a $(2 n+1)$-dimensional trans-Sasakian manifold which satisfies the condition $C_{0}(\xi, X) \cdot C_{0}(U, V) Z=0$, then by definition we have

$$
\begin{aligned}
0= & C_{0}(\xi, X) C_{0}(U, V) Z-C_{0}\left(C_{0}(\xi, X) U, V\right) Z \\
& -C_{0}\left(U, C_{0}(\xi, X) V\right) Z-C_{0}(U, V) C_{0}(\xi, X) Z .
\end{aligned}
$$

Using (2.22) in (6.1) we get

$$
\begin{aligned}
0= & R(\xi, X) \cdot C_{0}(U, V) Z+\left(\alpha^{2}-\beta^{2}-2\right)\left[g\left(X, C_{0}(U, V) Z\right) \xi\right. \\
& -\eta\left(C_{0}(U, V) Z\right) X-g(X, U) C_{0}(\xi, V) Z+\eta(U) C_{0}(X, V) Z \\
& -g(X, V) C_{0}(U, \xi) Z+\eta(V) C_{0}(U, X) Z-g(X, Z) C_{0}(U, V) \xi \\
& \left.+\eta(Z) C_{0}(U, V) X\right] .
\end{aligned}
$$

Taking inner product on both sides of (6.2) by $\xi$ and using (2.24), we obtain 


$$
\begin{aligned}
0= & g\left(R(\xi, X) \cdot C_{0}(U, V) Z, \xi\right)+\left(\alpha^{2}-\beta^{2}-2\right)\left[g\left(X, C_{o}(U, V) Z\right)\right. \\
& -\eta(X) \eta\left(C_{0}(U, V) Z\right)-g(X, U) \eta\left(C_{0}(\xi, V) Z\right) \\
& +\eta(U) \eta\left(C_{0}(X, V) Z\right)-g(X, V) \eta\left(C_{0}(U, \xi) Z\right) \\
& \left.+\eta(V) \eta\left(C_{0}(U, X) Z\right)-\eta(Z) \eta\left(C_{0}(U, V) X\right)\right] .
\end{aligned}
$$

Putting $U=\xi$ in (6.3) and using (2.22), (2.23) and (2.25), we get

$$
\begin{aligned}
0 & =g\left(R(\xi, X) \cdot C_{0}(\xi, V) Z, \xi\right) \\
& +\left(\alpha^{2}-\beta^{2}-2\right)[g(R(\xi, V) Z, X)+\eta(R(X, V) Z) \\
& \left.+\left(\alpha^{2}-\beta^{2}\right)\{\eta(Z) g(X, V)-2 \eta(X) g(V, Z)+\eta(V) g(X, Z)\}\right] .
\end{aligned}
$$

This implies that

$$
\begin{aligned}
& R(\xi, X) \cdot C_{0}(\xi, V) Z \\
& =\left(\alpha^{2}-\beta^{2}-2\right)\left[\left(\alpha^{2}-\beta^{2}\right)\{2 g(V, Z) X-g(X, V) Z\right. \\
& -g(X, Z) V\}-g(R(\xi, V) Z, X) \xi-R(X, V) Z] .
\end{aligned}
$$

From this it follows that the manifold is contact conformally semi-symmetric if the right hand side of (6.5) vanishes, i. e., if

$$
\begin{aligned}
0= & \left(\alpha^{2}-\beta^{2}\right)\{2 g(V, Z) X-g(X, V) Z-g(X, Z) V\} \\
& -g(R(\xi, V) Z, X) \xi-R(X, V) Z .
\end{aligned}
$$

This completes the proof of the theorem.

\section{An Example of a 3-dimensional Trans-Sasakian Manifold}

Let us consider a 3 -dimensional manifold $M=\left\{(x, y, z):(x, y, z) \in \mathbb{R}^{3}\right\}, z \neq 0$, where $(x, y, z)$ are the standard coordinates in $\mathbb{R}^{3}$. We choose the vector fields

$$
e_{1}=e^{z}\left(\frac{\partial}{\partial x}+y \frac{\partial}{\partial z}\right), e_{2}=e^{z} \frac{\partial}{\partial y}, e_{3}=\frac{\partial}{\partial z}
$$

which are linearly independent at each point of $M$. Now we define a semi-Riemannian metric $g$ on $M$ as

$$
\begin{gathered}
g\left(e_{1}, e_{3}\right)=g\left(e_{2}, e_{3}\right)=g\left(e_{1}, e_{2}\right)=0, \\
g\left(e_{1}, e_{1}\right)=g\left(e_{2}, e_{2}\right)=g\left(e_{3}, e_{3}\right)=1 .
\end{gathered}
$$

Let $\eta$ be a 1 -form defined by $\eta(Z)=g\left(Z, e_{3}\right)$ for any vector field $Z \in M$ and $\varphi$ be a $(1,1)$ tensor field defined by 


$$
\varphi\left(e_{1}\right)=e_{2}, \varphi\left(e_{2}\right)=-e_{1}, \varphi\left(e_{3}\right)=0 \text {. }
$$

The linearity property of $\varphi$ and $g$ yields that

$$
\eta\left(e_{3}\right)=1, \varphi^{2}(Z)=-Z+\eta(Z) e_{3}, g(\varphi Z, \varphi U)=g(Z, U)-\eta(Z) \eta(U)
$$

for any $Z, U \in M$.

If we take $e_{3}=\xi$ in (7.5), $(\varphi, \xi, \eta, g)$ defines an almost contact metric structure on $M$.

By the definition of Lie bracket and (7.1) we have

$$
\begin{aligned}
{\left[e_{1}, e_{2}\right]=e_{1} e_{2}-e_{2} e_{1} } & =e^{z}\left(\frac{\partial}{\partial x}+y \frac{\partial}{\partial z}\right) e^{z} \frac{\partial}{\partial y}-e^{z} \frac{\partial}{\partial y}\left(e^{z} \frac{\partial}{\partial x}+y e^{z} \frac{\partial}{\partial z}\right) \\
& =y e^{z} e_{2}-e^{2 z} e_{3} .
\end{aligned}
$$

Proceeding same way we obtain $\left[e_{2}, e_{3}\right]=-e_{2}$ and $\left[e_{1}, e_{3}\right]=-e_{1}$. Thus we have

$$
\left[e_{1}, e_{2}\right]=y e^{z} e_{2}-e^{2 z} e_{3},\left[e_{2}, e_{3}\right]=-e_{2},\left[e_{1}, e_{3}\right]=-e_{1} \text {. }
$$

Let $\nabla$ be the Levi-Civita connection with respect to $g$ then we have the Koszul's formula

$$
\begin{aligned}
2 g\left(\nabla_{X} Y, Z\right) & =X g(Y, Z)+Y g(Z, X)-Z g(X, Y) \\
& +g([X, Y], Z)-g([Y, Z], X)+g([Z, X], Y) .
\end{aligned}
$$

By the use of (7.2), (7.3) and (7.6), (7.7) yields

$$
\left\{\begin{array}{l}
\nabla_{e_{1}} e_{3}=-e_{1}+\frac{1}{2} e^{2 z} e_{2}, \quad \nabla_{e_{1}} e_{2}=\frac{1}{2} e^{2 z} e_{3}, \nabla_{e_{1}} e_{1}=e_{3}, \\
\nabla_{e_{2}} e_{3}=-e_{2}-\frac{1}{2} e^{2 z} e_{1}, \quad \nabla_{e_{2}} e_{2}=e_{3}+y e^{z} e_{1}, \nabla_{e_{2}} e_{1}=-y e^{z} e_{2}+\frac{1}{2} e^{2 z} e_{3}, \\
\nabla_{e_{3}} e_{3}=0, \quad \nabla_{e_{3}} e_{2}=-\frac{1}{2} e^{2 z} e_{1}, \nabla_{e_{3}} e_{1}=\frac{1}{2} e^{2 z} e_{2},
\end{array}\right.
$$

In view of (2.6), (7.2), (7.3) and (7.4) we have

$\nabla_{e_{1}} \xi=\beta e_{1}-\alpha e_{2}, \nabla_{e_{2}} \xi=\alpha e_{1}+\beta e_{2}$ and $\nabla_{e_{3}} \xi=0$ for $e_{3}=\xi$. Comparing these equations with

(7.8) (first column), we get $\alpha=-\frac{1}{2} e^{2 z}$ and $\beta=-1$.

Again, by virtue of (2.7) and $\left(\nabla_{X} \eta\right) Y=\nabla_{X} \eta(Y)-\eta\left(\nabla_{X} Y\right)$ we obtain

$$
\left(\nabla_{e_{1}} \eta\right) e_{1}=\beta=-1,\left(\nabla_{e_{2}} \eta\right) e_{1}=\alpha=-\frac{1}{2} e^{2 z},\left(\nabla_{e_{3}} \eta\right) e_{1}==0 \text {. }
$$

Thus from above calculation the conditions (2.6) and (2.7) are satisfied and the structure $(\varphi, \xi, \eta, g)$ is a trans-Sasakian structure of type $(\alpha, \beta)$ where $\alpha=-\frac{1}{2} e^{2 z}$ and $\beta=-1$. Consequently $M^{3}(\varphi, \xi, \eta, g)$ is a trans-Sasakian manifold. 


\section{References}

[1] A. Gray, and L. M. Harvella, Ann. Mat. Pura. Appl., 123(4)(1980) 35-58. http://dx.doi.org/10.1007/BF01796539

[2] J. A. Oubina, Publ. Math. Debrecen, 32(1985) 187-193.

[3] D. E. Blair, Contact manifolds in Riemannian geometry, Lecture Notes in Math., Vol. 509, Springer-Verlag, Berlin, (1976).

[4] D. E. Blair, Riemannian geometry of contact and sympletic manifolds, Birkhauser Boston, 2002.

http://dx.doi.org/10.1007/978-1-4757-3604-5

[5] K. Kenmotsu, Tohoku Math. J., 24(1972) 93-103.

[6] U. C. De and M. M. Tripathi, Kyungpook Math. J., 43(2003) 247-255. http://dx.doi.org/10.2748/tmj/1178241594

[7] R. J. Shah, Kathmandu Univ. J. of Sci., Eng. and Tech., 8(1)(2012) 81-87.

[8] J. C. Jeong, J. D. Lee, G. H. Oh. and J. S. Pak, Bull. Korean Math. Soc., 27(1990) 133-142.

[9] H. Kitahara, K. Matsuo and J. S. Pak, Bull. Korean Math. Soc., 27(1990) 27-30.

[10] J. C. Jeong and J. K. Park, Comm. Korean Math. Soc., 8(1993) 689-697.

[11] J. S. Pak, J. C. Jeong and W. T. Kim, J. Korean Math. Soc. 28(1991) 267-274. 